\title{
Erratum to: Management of HCV infection in the penitentiary setting in the direct-acting antivirals era: practical recommendations from an expert panel
}

\author{
Roberto Ranieri ${ }^{1,2}$ • Giulio Starnini $i^{2,3} \cdot$ Sergio Carbonara ${ }^{2,4} \cdot$ Emanuele Pontali $^{2,5}$ - Guido Leo ${ }^{2,6}$. \\ Antonio Romano $^{7} \cdot$ Sandro Panese $^{8} \cdot$ Roberto Monarca $^{2,9} \cdot$ Tullio Prestileo $^{10}$ - Giorgio Barbarini ${ }^{11,12}$. \\ Sergio Babudieri ${ }^{2,13} \cdot$ on behalf of the SIMSPe Group
}

Published online: 8 February 2017

(C) Springer-Verlag Berlin Heidelberg 2017

\section{Erratum to: Infection}

\section{DOI 10.1007/s15010-016-0973-0}

The original version of this article unfortunately contained a mistake. The given names and family names of all authors were transposed. The correct names are as shown above.

The original article has been corrected.

The online version of the original article can be found under doi:10.1007/s15010-016-0973-0.

Sergio Babudieri

babuder@uniss.it

1 Azienda Ospedaliera Santi Paolo e Carlo Penitentiary Infectious Diseases Unit, University of Milan, Milan, Italy

2 SIMSPe-Italian Society for Penitentiary Medicine and Healthcare, v.le San Pietro35/b, 07100 Sassari, Italy

3 U.O.C. Medicina protetta-Malattie infettive, Viterbo, Italy

4 Clinic of Infectious Diseases, University of Bari, Bari, Italy

5 Infectious Disease Unit, Ospedale Galliera, Genoa, Italy

6 Infectious Disease Unit, Ospedale Amedeo di Savoia, Turin, Italy
Cisanello Hospital, Pisa, Italy

8 Ospedale dell'Angelo Malattie infettive e Tropicali, Venice, Italy

9 Ospedale Belcolle, Viterbo, Italy

10 ARNAS, Ospedale Civico-Benfratelli Palermo, Palermo, Italy

11 Malattie Infettive e Tropicali IRCCS San Matteo, Università di Pavia, Pavia, Italy

12 Case Circondariali di Pavia, Voghera E Vigevano, Italy

13 Infectious Disease Unit, University of Sassari, Sassari, Italy 